\title{
A Physical Method to Incorporate Parasitic Elements in a Circuit Simulator Based on the Partial Inductance Concept
}

\author{
B.H. Evenblij \\ TNO Prins Maurits Laboratory \\ P.O. Box 4, 2280 AA Rijswijk, The Netherlands \\ Email: evenbly@pml.tno.nl
}

Delft University of Technology, Electrical Power Processing group

PO Box 5031, 2600 GA Delft, The Netherlands

Email: J.A.Ferreira@ITS.TUDelft.NL

physical perspective. A method is established to define and measure node-voltages in an environment where the voltage between two points is undefined because of a time-varying magnetic field. In order to get at calculation results however, a fine theoretical definiton is not enough: one has to have a practical way to calculate modelparameters. The concept of partial inductances is introduced at this point as well as a certain stylising of the circuits. The process of the modeling a realistic circuit is described in order to make clear how the different concepts introduced thus far fit together. After that a more sizeable circuit is presented that has been used to validate the modeling. The validation discussion concludes the paper.

\section{INTRODUCTION}

The quest for the ideal switch has been going on for quite a while now and has led to remarkable results. The more this ultimate goal is approached however, the more the non-ideal properties of the environment of the switch manifest themselves. As there is no possibility of improving conductors as is the case with semiconductors, the features of the conductors have to be accepted. The only thing the engineer can do about parasitic features of surrounding circuit of the switches is modeling them properly and designing the geometry of the circuit in such a way that the semiconductors (and other components) won't be stressed beyond their specs.

This modeling can be done using either FE-techniques (Finite Element) or the LE-approach (Lumped Element). In general one can say that FE-analysis produces vast amounts of detailed information, but only at one point of time. The abundance of information supplied by FE-analysis can be unfavourable. Moreover trends are only to be noticed after numerous simulations that allow for plotting characteristic features as a function of input parameters. LE-analysis delivers information as a function of time, but this information is not always accurate enough. Because the LEmodeling implies the analytical solution of a part of the (simplified) initial problem, one ends up with much less system parameters, that make for instance the investigation of parameter-sensitivity much more easy. The object of this paper is to develop a LE-model that approaches FE-detail and accuracy.

The paper is organised as follows. In order to be able to incorporate parasitic elements in a circuit simulation, some understanding of the models and how the circuit simulator uses them is necessary. This topic is briefly discussed in the paragraph 2. This discussion allows for looking at how to model parasitic elements in the next paragraph. After that inductive and resistive parasitics are looked at from a

\section{PRinCiple of OpERATION Of CiRCUIT Simulators.}

The considerations hereafter apply in principle to any circuit simulator based on the solving of Kirchhoffs Current Law. As Saber ${ }^{\mathrm{TM}}$ is used for the simulation, the syntax in the examples is taken from this simulator.

Consider the simple case of a circuit consisting of a current source and a resistor in Fig. 1. The crucial line in the code of the model of the resistor is:

$i: \quad v(a)-v(b)=i R$

Its meaning can be stated as: "Find $i$ under the condition that the difference between terminal voltages equals $R$ times the value of $i$ ". What the simulator does is writing down the $\mathrm{KCL}$ for each node (except 0 -node), which is in this case:

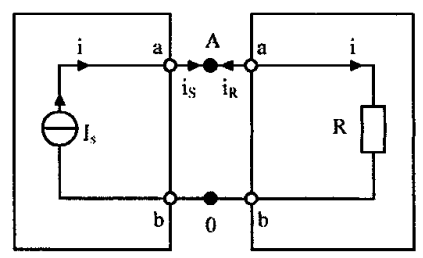

Fig. 1. Simulation of circuit with current source and resistor

$i_{R}+i_{s}=0$

Next it will substitute for all the unknown currents expressions that contain the dependence of the currents from the node-voltages. In this case the result is:

$-(v(A)-v(0)) \frac{1}{R}+I_{S}=0$

Mark the distinction between internal nodes, currents and voltages and external nodes, currents and voltages. In the 
next stage the simulator will for each node change the voltages until for all nodes the total sum of currents is sufficiently equal to zero. This search for node voltages is done by a numerical iteration method as for instance NewtonRapson.

The central issue is that in the model description of each component an implicit or explicit relation is given from which the simulator can derive the dependency of all the currents from the node voltages.

\section{TWO WAYS OF IMPLEMENTING PARASITIC SERIES RESISTANCE}

Consider the circuit of Fig. 2. In this figure a simple circuit containing a voltage source and a resistor are depicted. Both the voltage source and the resistor are represented as ideal components. Their respective models are as follows:

Voltage source: $i: \quad v(a)-v(b)=U_{S}$

Resistor: $\quad i: \quad v(a)-v(b)=i R$

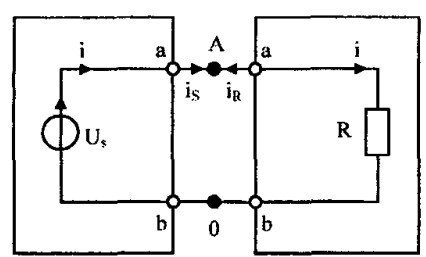

Fig. 2. Simulation of circuit with voltage source and resistor

The values of $U_{S}$ and $R$ are parameters of the models. They are to be seen as constants with a known value.

Suppose we want to add to the model the effects of the wiring resistance. We can do this in two ways. The first way is to edit the model of both the voltage source and of the resistor. This results in adapted model descriptions:

Voltage source: $i: v(a)-v(b)=U_{s}-i R p_{s a}-i R p_{s b}$

Resistor: $i: \quad v(a)-v(b)=i R+i R p_{r a}+i R p_{r b}$

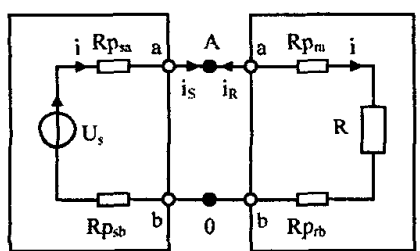

Fig. 3. Parasitics integrated in component models

Where $R p_{s a}$ and $R p_{s h}$ are the values of the parasitic resistors of the source cables of terminal $a$ and $b$; and $R p_{r a}$ and $R p_{r b}$ are the values of the parasitic resistors of the resistor cables of terminal $a$ and $b$. The resulting models are depicted in Fig. 3.

The other way of incorporating the series resistors of the wiring however is by adding a model that contains only the resistive effects of the wiring. See Fig. 4. The description of the wiring model in Fig. 4 is:

$$
\begin{array}{ll}
i_{1}: & v(a)-v(c)=i_{1} R p_{s a}+i_{1} R p_{r a} \\
i_{2}: & v(d)-v(b)=i_{2} R p_{s b}+i_{2} R p_{r b}
\end{array}
$$

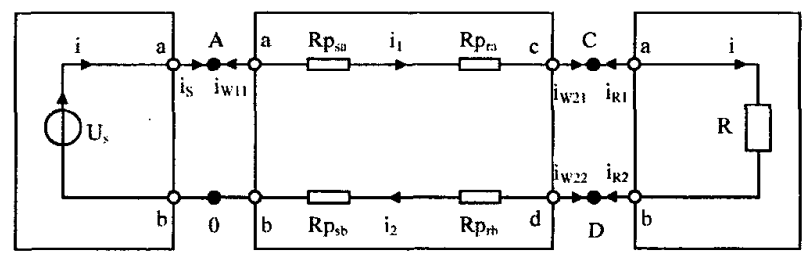

Fig. 4. Parasitics modeled in a separate wiring model

Observe it is possible to model other parasitic effects as well in both ways. Suppose the resistor has a small value of series inductance. It is possible to add this impedance to the model of the resistor itself or to attribute this series inductance to the model containing the wiring parasitics. In the case of an inductance however there are two major differences. There exists, strictly spoken, no such thing as an inductance of a resistor: inductances only apply to closed loops. But if one had a concept to attribute an inductance to a non-closed piece of wire, this element could be modeled in both ways just described. The problem of attaching inductances to branches instead of loops will be addressed further on in the paper.

The other difference is, that the voltage of a resistor depends solely on the current flowing through the resistor, whereas the voltage of an inductor in principle depends on all the currents in the circuit. This dependency can be modeled by adding current-controlled voltage-sources in series with the parasitic impedances. This addition however does not affect the argument anywhere in this paragraph.

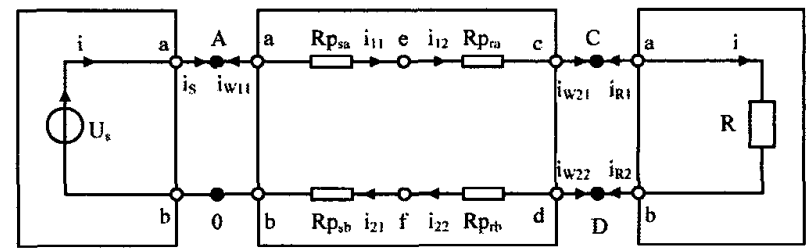

Fig. 5. Extra internal nodes " $\mathrm{e}$ " and " $\mathrm{f}$ " in wiring model

Observe also that one has to be careful to interpret the calculated values of the node voltages. In the case of Fig. 3, the node voltages correspond to the voltages that can be measured at the physical terminals of the source and the resistor. In Fig. 4 the calculated node voltages might not be available for measurement, because they represent the node voltages of the ideal parts of the source and resistor. In order to generate in the simulation measurable data as well one might add two internal nodes $e$ and $f$ to the model of Fig. 4, as is done in the model of Fig. 5. The model description becomes then: 


$$
\begin{array}{ll}
i_{11}: & v(a)-v(e)=i_{11} \text { Rpsa } \\
i_{12}: & v(e)-v(c)=i_{12} \text { Rpra } \\
i_{21}: & v(f)-v(b)=i_{21} \text { Rps } b \\
i_{22}: & v(d)-v(f)=i_{22} \text { Rprb }
\end{array}
$$

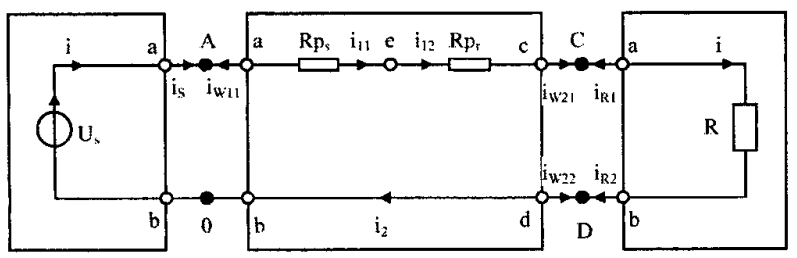

Fig. 6. Parasitics modeled as one element for each component

A last point to be made in this paragraph concerns the division of the parasitic elements of one component into two separate ones in the model of the wiring. Although this is suggested by the fact that components have in general two more or less equally shaped leads, there is no real argument against modeling the total parasitic resistance of a component in the wiring model as one resistor. This is depicted in Fig. 6.

In looking at Fig. 6 , one sees that the node $b$ and $d$ have become identical to node $f$ in Fig. 5. This allows for leaving out one of them. As a result one gets the circuit of Fig. 7. In this case there are no currents flowing to node $b$ from nodes within wiring model. As a consequence node $b$ could be wholly left out of the wiring model. In practice however it turns out to be more simple for the user of the model, if these nodes are retained within the wiring model, although they only serve as a collecting node for purely external currents.

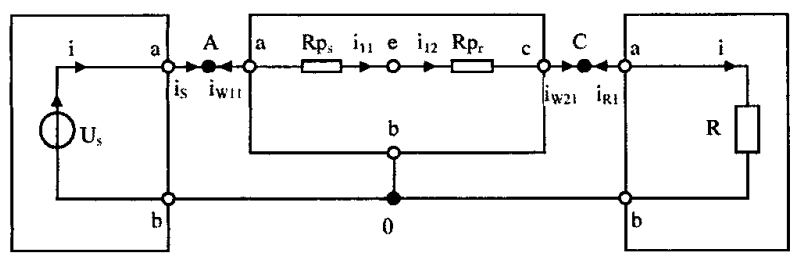

Fig. 7. Combination of short-circuited nodes into one

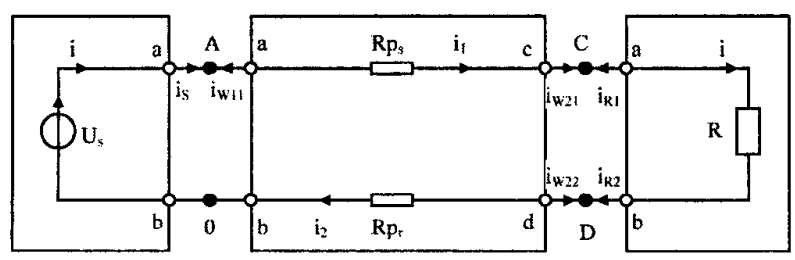

Fig. 8. Intemal node disappears after rearrangement of parasitic elements

Observe that for each branch there are two external connection points, in order to connect the models of the components to the wiring model. In addition to these often an internal node is present like $e$ in Fig. 7. If one had chosen for a different combination of the two parasitic resistances the situation of Fig. 8 would have resulted. Here no internal nodes are present. Both situations can occur in the program.

\section{Physical meaning of terminal voltages}

The current distribution in wires and components constitutes a magnetic field. The time-derivative of this field gives rise to an electric field that causes inductive voltages. As a consequence the voltage of a given point within the circuit and its immediate neighbourhood is no longer defined. The circuit simulator calculates however for each time-step the nodevoltages, under the condition that the total current for each node is zero. If one wants to make use of a circuit simulator two things require attention:

- to find an adapted and physically sound definition of terminal voltages

- to identify the way to implement this definition in the circuit simulator

In the quasistatic approximation the choice of two points $a$ and $b$ uniquely determines the voltage between these points, independently of the path chosen:

$V_{b a}=-\int_{a}^{b} \vec{E} \bullet \vec{\tau} d S$

The voltage of a given point can moreover be established by chosing an arbritary value for the voltage in one point, that is in practice often the "zero" of the circuit.

In order to retain the distinctness of the voltage the path along which the integral is taken has to be specified. This is illustrated in Fig. 9.

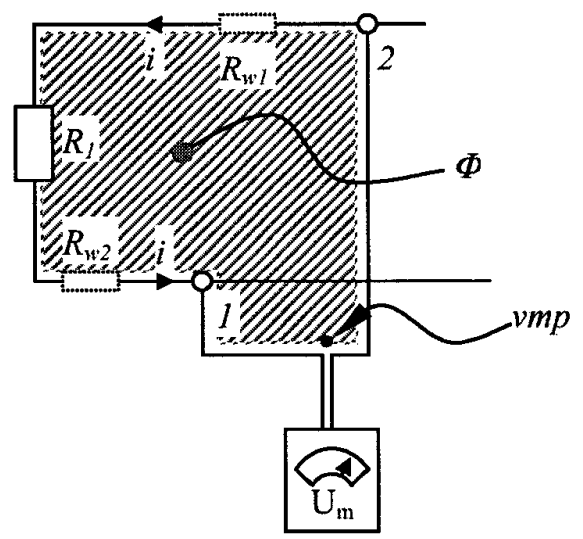

Fig. 9. Measurement of voltage between nodes 1 and 2 .

If resistor and wiring can be supposed ideal :

$V_{21}=i_{1} R_{1}$ 


$$
V_{21}=i_{1} R_{1} \div i_{1} R_{w 1}+i_{i} R_{w 2}
$$

If the inductive field plays an important role as well:

$$
V_{21}=i_{1} R_{1}+i_{1} R_{w 1}+i_{i} R_{w 2}-\partial_{t} \Phi
$$

But this expression is not determined, unless (1) the position of the voltagemeter and (2) the connecting wires to node 1 and 2 are defined. In Fig. 9, the measuring wires come together at the black dot, indicated with vmp. This is the virtual measuring point. An imaginary very small measuring device would at that point register the same voltage as a real measuring device at some distance, if its measuring wires catch up no flux between the vmp and the voltagemeter. So in prescribing a vmp and a connection path between all of the nodes of the circuit and the vmp one establishes a unique definition for all the branchvoltages. Because of the fact that a closely twisted pair of measurement wires from the vmp to the measuring device allows for any distance between circuit and voltagemeter, a feasible way to measure these voltages is provided as well.

Observe that the flux is often linearly dependent on all the branchcurrents in the circuit. If we take this linearity as a valid assumption, the last expression can be written as:

$$
\begin{aligned}
V_{21} & =i_{1} R_{1}+i_{1} R_{w 1}+i_{i} R_{w 2}+a_{1} \partial_{t} i_{1}+a_{2} \partial_{t} i_{2}+\ldots \\
& +\alpha_{N} \partial_{t} i_{N}
\end{aligned}
$$

Where one or more $\alpha_{i}$ may be zero.

Consider the case that one has for each branch an expression like (14) available. It is a well-known fact from circuit theory that this set of expressions is the complete statement of the circuit problem. If the user supplies for each branch a statement like (14), the simulator will solve the nodevoltages, and these nodevoltages do physically make sense.

The calculation of the values of $\alpha_{i}$ is the subject of the next paragraph.

\section{Calculation of parasitic inductances}

The object of this paragraph is to establish a practical method to calculate the flux in (13).

It is possible to take the following procedure. Chose all branchcurrents in the circuit except one equal to zero. Calculate the magnetic field due to this current on the surface of interest, i.e. the shaded area in Fig. 9. This is done by making use of Biot-Savart's law. Integrate the flux-density over this area and divide by the magnitude of the current in the flux-generating branch. Repeat this process for each of the branches finding in each case the value of $\alpha$ pertaining to that branch. As the flux-receiving area is in general a 3D surface, this is a rather difficult task, because it involves numerical integrations to three variables that will in general be mutually dependent.
There is however a much more practical way in calculating these values, which is based upon the concept of partial inductances of rectangular shapes [1]. This method in the first place defines partial inductances between branches instead of loops. And, secondly, it supplies a closed form solution of the partial inductance between two rectangular shaped conductors carrying both homogeneous current. So if one is able to describe (or approximate) the circuit in terms of rectangular shaped components and wires, this concept provides closed form formulas to calculate the abovementioned $\alpha \dot{s}$. This method is adopted here and consists basically of three steps:

1.Attach the flux of each loop to the surrounding contour, i.e. attach partial fluxes to the branch and to the two measurement wires.

2. Stylise every branch and its components as well as all the measurement wires in one or more rectangular bars.

3. Make use of the closed form solution for the mutual partial inductance between two rectangular shaped conductors.

In order to make clear what is to be done, the concept of inductances is briefly reviewed here and then it is shown how the concept of partial inductances is developed from this.

Consider at first the situation of two current loops, $p$ and $q$, that have currents $I_{p}$ and $I_{q}$, and current densities $J_{p}$ and $J_{q}$. See Fig. 10. Along with $J_{p}$ and $J_{q}$ are defined the current density distributions, indicated with the superscript ${ }^{(1)}$ :

$\vec{J}_{p}^{(1)}=\frac{\vec{J}_{p}}{I_{p}}, \quad \vec{J}_{q}^{(1)}=\frac{\vec{J}_{q}}{I_{q}}$

The general expression for the flux $\phi_{q}$ connected with a loop $p$ due to a current loop $q$ is by definition:

$\phi_{p q}=\iiint_{V_{p}} \vec{A}_{q} \bullet \vec{J}_{p}^{(1)} d V$

where $\vec{A}_{q}$ is the vector potential due to the current in loop $q$, and is defined by

$\nabla \times\left(\mu^{-1} \nabla \times \vec{A}_{q}\right)=\vec{J}_{q}$

If relative permeability is 1 everywhere $\vec{A}_{q}$ can be expressed as:

$$
\vec{A}_{q}\left(\vec{r}^{\prime}\right)=\iiint_{V} \frac{\mu_{0} \vec{J}_{q}^{(1)}(\vec{r})}{4 \pi R} d V
$$

This leads to the general expression for mutual inductance:

$L_{p q}=\iiint_{V_{p}} d V^{\prime} \iiint_{V_{q}} \frac{\mu_{0} \vec{J}_{p}^{(1)}\left(\vec{r}^{\prime}\right) \bullet \vec{J}_{q}^{(1)}(\vec{r})}{4 \pi R} d V$ 


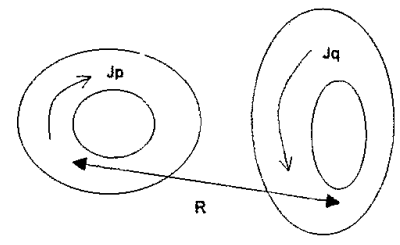

Fig. 10. Mutual inductance between to loops $p$ and $q$

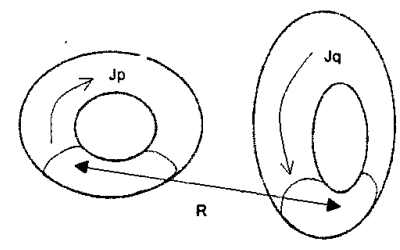

Fig. 11. Partial inductance between to parts of the loops $p$ and $q$

In looking at this last equation one concludes that for the calculation of the inductances only the volume of the areas where the current is flowing (i.e. in practical circuits in the connecting wiring and in the components), have to be taken into account.

Now the concept of partial inductances is introduced. This is taken from the literature [1]. A conventional inductance is the proportionality factor between a flux linked with an area or its bordering loop, due to a currentloop. Basically between a flux-generating current loop and a flux-receiving loop. The concept of partial inductances defines a way to associate a flux linked with a branch with a branch-current. In Fig. 11 the definition of the partial inductance between to branches is illustrated:

$$
L p_{p i q j}=\iiint_{V p i} d V^{\prime} \iiint_{V q j} \frac{\mu_{0} \vec{J}_{p}^{(1)}\left(\vec{r}^{\prime}\right) \bullet \vec{J}_{q}^{(1)}(\vec{r})}{4 \pi R} d V
$$

So the same expression as for the conventional inductances is taken, but the integration is performed only on the parts $V_{p}$ and $V_{q j}$ of the respective loops $p$ and $q$.

In terms of the last paragraph we have now a theoretical basis for the flux linked with a branch or a measurement wire on the one hand (that is on the flux-receiving part) and a branchcurrent elsewhere in the circuit on the other hand (that is on the flux-generating part). So the first step is taken: a method is defined break down the flux of the loop, into (partial) fluxes related to the branch and to each of the two measurement wires respectively

If $V_{p i}$ and $V_{q i}$ satisfy certain geometrical features this integral can partially or wholly be integrated. In the cases of parallel and rectangular bars as depicted in Fig. 12, an analytical solution has been published by Love and Hoer [2] that has been used by Ruehli in his article on partial inductances [1].

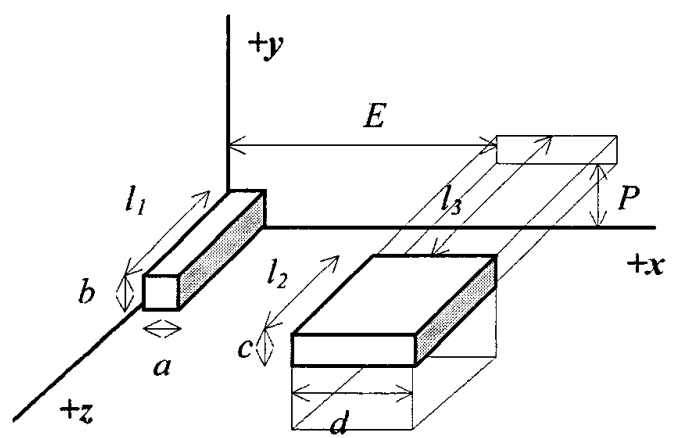

Fig. 12. general geometry for which a closed form solution of partial coupling is known

The second thing to be done in order to acquire the values of the parasitic inductances, is to stylise every branch and its components as well as all the measurement wires in one or more rectangular bars. Then for each branch and its components and for each measurement wire the partial inductance with all the (other) branches can be calculated, using the aforementioned analytical solution. The proces of modeling a physical branch is the subject of the next paragraph.

In order to show which steps have been taken (13) is rewritten, splitting up the flux into three partial fluxes:

$$
\begin{gathered}
V_{21}=i_{1} R_{1}+i_{1} R_{w 1}+i_{i} R_{w 2} \\
\partial_{t} \phi p_{m 1}+\partial_{t} \phi p_{m 2}+\partial_{t} \phi p_{b 1}
\end{gathered}
$$

Where the index $m$ stands for measurement wire and $b$ for branch. Then these partial fluxes are expressed in partial inductances and branchcurrents:

$$
\begin{aligned}
& V_{21}=i_{1} R_{1}+i_{1} R_{w 1}+i_{i} R_{w 2}+ \\
& L p_{m 1, b 1} \partial_{t} i_{1}+L p_{m 1, b 2} \partial_{t} i_{2}+\ldots+L p_{m 1, b N} \partial_{t} i_{N}+ \\
& L p_{m 2, b 1} \partial_{t} i_{1}+L p_{m 2, b 2} \partial_{t} i_{2}+\ldots+L p_{m 2, b N} \partial_{t} i_{N}+ \\
& L p_{b 1, b 1} \partial_{t} i_{1}+L p_{b 1, b 2} \partial_{t} i_{2}+\ldots+L p_{b 1, b N} \partial_{t} i_{N}
\end{aligned}
$$

All these partial inductances $L p$ can be calculated purely on the basis of the stylised geometrical information of the wiring and of the components, with the aid of the formulas by Love and Hoer.

Equation (22) readily fits in with the concept of putting parasitic features into a comprehensive wiring model, to which the ideal component models are to be attached. The first item in the righthand side: $i_{1} R_{1}$ will be put in the ideal model. The remainder is to be put up in the model of the wring: 


$$
\begin{aligned}
& i_{1} R_{w 1}+i_{i} R_{w 2}+ \\
& L p_{m 1, b 1} \partial_{t} i_{1}+L p_{m 1, b 2} \partial_{t} i_{2}+\ldots+L p_{m 1, b N} \partial_{t} i_{N}+ \\
& L p_{m 2, b 1} \partial_{t} i_{1}+L p_{m 2, b 2} \partial_{t} i_{2}+\ldots+L p_{m 2, b N} \partial_{t} i_{N}+ \\
& L p_{b 1, b 1} \partial_{t} i_{1}+L p_{b 1, b 2} \partial_{t} i_{2}+\ldots+L p_{b 1, b N} \partial_{t} i_{N}
\end{aligned}
$$

\section{EXAMPLE OF MODELING A PHYSICAL CIRCUIT}

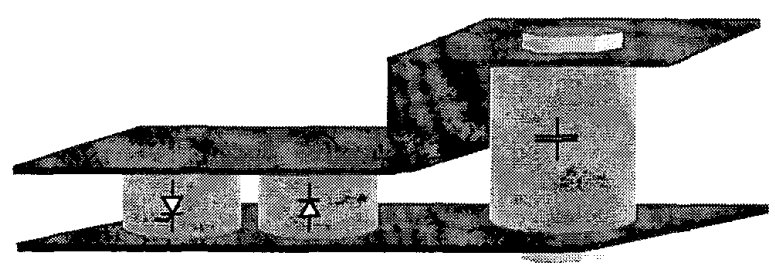

Fig. 13. Physical circuit to be simulated

Consider Fig. 13. In this figure a simple but realistic piece of power electronics is depicted. The symbols on the components suggest the circuit of Fig. 14.

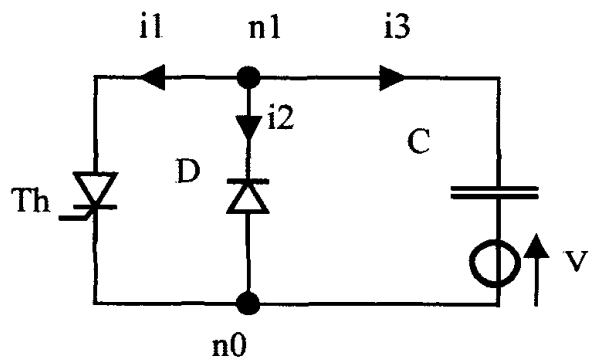

Fig. 14. Circuit diagram of physical circuit

The initial condition of the voltage on the capacitor is accounted for by the external voltage source. Mark the definition of the branch currents as well as of the nodes.

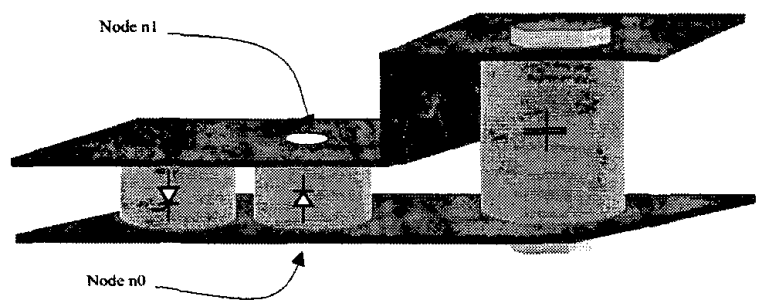

Fig. 15. Identification of physical areas corresponding to nodes

The first thing to be done is to chose in Fig. 13 physical spots that correspond to the nodes in Fig. 14. These spots are the (small) areas where one can put a probe on, in order to measure the node voltage. In this example only two nodes are chosen, see Fig. 15.

Other and especially more nodes can be chosen. This requires a lengthy discussion, which is beyond the scope of this paper, and will be left out of consideration here.

Secondly, again in Fig. 13, the physical (3D-)regions where the branch-currents in Fig. 14 flow, are to be identified.

Thirdly one has to stylise in these current-regions the wiring and the components in rectangular blocks of homogeneous current that do approximate the real currentdensity distribution. Not only the blocks are to be chosen, but the current-direction as well. This is indicated in Fig. 16.

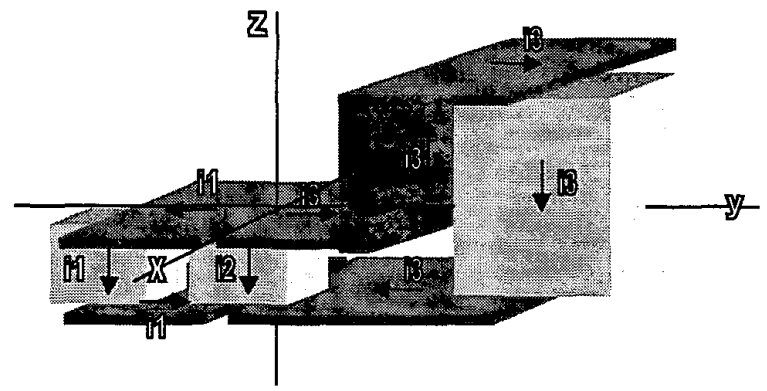

Fig. 16. Approximation of current distribution by rectangular regions of homogeneous current

Now a system of coordinates is chosen, with reference to which the stylised current blocks can be characterized. For each block the startingpoint $\left(x_{0}, y_{0}, z_{0}\right)$ and endpoint $\left(x_{i}, y_{l}, z_{l}\right)$ of the centre-line of the current are denoted as well as the width and the thickness of the block, see Table I. Also in Table I, the occurrence of the components is indicated.

Table I. Geometrical data of current blocks

\begin{tabular}{|r|r|r|r|r|r|r|r|r|r|r|}
\hline Branch block & width & $\begin{array}{c}\text { thick- } \\
\text { ness }\end{array}$ & $\mathbf{x 0}$ & $\mathbf{y 0}$ & $\mathbf{z 0}$ & $\mathbf{x 1}$ & $\mathbf{y 1}$ & $\mathbf{z 1}$ & $\begin{array}{c}\text { Compo- } \\
\text { nents }\end{array}$ \\
\hline 1 & 1 & 50 & 1 & 0 & 0 & 0 & 0 & -60 & 0 & \\
\hline 1 & 2 & 40 & 40 & 0 & -60 & 0 & 0 & -60 & -30 & Th \\
\hline 1 & 3 & 50 & 1 & 0 & -60 & -30 & 0 & 0 & -30 & \\
\hline 2 & 4 & 40 & 40 & 0 & 0 & 0 & 0 & 0 & -30 & 0 \\
\hline 3 & 5 & 50 & 1 & 0 & 0 & 0 & 0 & 60 & 0 & \\
\hline 3 & 6 & 50 & 1 & 0 & 60 & 0 & 0 & 60 & 50 & \\
\hline 3 & 7 & 50 & 1 & 0 & 60 & 50 & 0 & 110 & 50 & \\
\hline 3 & 8 & 70 & 70 & 0 & 110 & 50 & 0 & 110 & -30 & \\
\hline 3 & 9 & 50 & 1 & 0 & 110 & -30 & 0 & 0 & -30 & \\
\hline
\end{tabular}

The data from Table I are the input data of the program, that generates the simulation model of the wiring. In order to enable the user to check whether he has entered the correct data, the program also generates a picture of the wire-model i.e. the centre lines of currents in wiring and components, see 
Fig. 17. The program generates automatically internal and external nodes. The picture of the wire-model enables the user to identify their position. Comparing Fig. 17 and Fig. 14 shows that node $n I$ corresponds to in 1 and node $n 0$ tot ex2.

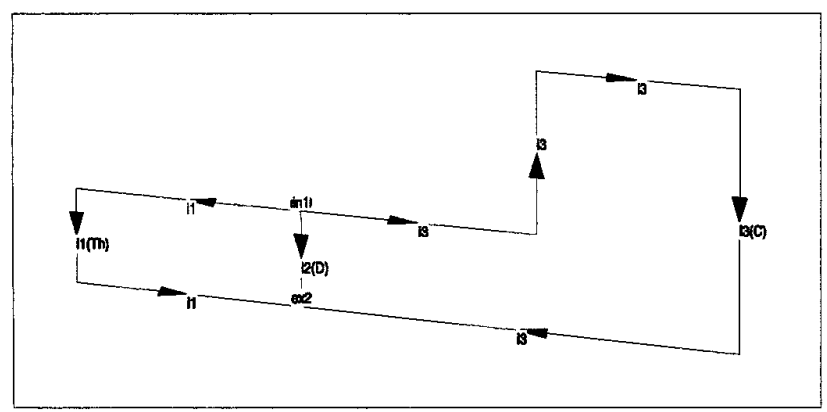

Fig. 17. Wire model, i.e. centre line of currents in wiring and components

The simulationmodel generated by the program is a Saber ${ }^{\mathrm{TM}}$ template and reads as in Fig. 18. The template has four external connection points: $p 1, p_{2}, p_{3}$ and ex2. Remember that (in paragraph 3) it was decided that for each branch a pair of connection-points is to be made available. Each of these pairs defines an external voltage. In this case of three branches, they are vex 1 , vex 2 and vex3. By definition the side where positive current is flowing out of the template into the connected component is called p1, p2 and p3 respectively. The other external connection-point (i.e. ex2) can either be gathered from Fig. 17 or from the template:

$v \operatorname{ex} 1=v(p 1)-v(\operatorname{ex} 2)$

vex $2=v(p 2)-v(e x 2)$

vex $3=v(p 3)-v(e \times 2)$

For each branch there is in this template a crucial line of code, take for instance branch 2:

i2: $\operatorname{vin} 2=\mathrm{d} \_b y \_d t(-\mathrm{fpm} 1+\mathrm{fpm} 2+\mathrm{fp} 2)+v e x 2+$ i2* $\quad 0.3187 \overline{1 e}-6$

It states that the total branch-voltage vin2 consists of three parts:

- the time derivative of the partial flux fpm (connected with measurement wire 1), fpm2 (connected with measurement wire 2) and $\mathrm{fp}_{2}$ (connected with the branch itself)

- The voltage vex 2 due to some possible external component which can be connected to the template so that it carries the branch-current. Remember the concept we adopted to model the parasitics of the wiring as a seperate model, to which the ideal components are to be connected.

- The resistive voltage drop along the branch: i2 $20.31871 e-6$.

The correspondence between this line of code and (21) is easily apprehended.

Because the program choses automatically the point $(x, y, z)=(0,0,0)$ as the virtual measurement point, the measurement wire from internal node inl (coinciding with $(0,0,0))$ is degenerate. This is reflected in the line of code that expresses the way the partial flux connected with this measurement-wire, depends on the branch-currents: fpmi $=(0.0000 * i 1+0.0000 * i 2+0.0000 * i 3) * 1 e-9$ The other measurment-flux has of course a non-zero coupling with each of the three branch-currents:

fpm2 $=(-1.4709 * i 1+3.9206 * i 2+-0.0171 * i 3) * 1 e-9$

The condition of homogeneous current will not always be sufficiently satisfied, due to skinning. Non-homogeneous current in the first place affects selfinductance and in a considerably smaller degree mutual inductances. The factor Fsk reduces the selfinductances. If skinning is complete, no internal field will exist in the conductors, resulting in a reduction to some $90 \% .80 \%$ of selfinductance for most practical situations. Although theoretically this is not a very strong feature of the model it turns out to be very handy in practice.

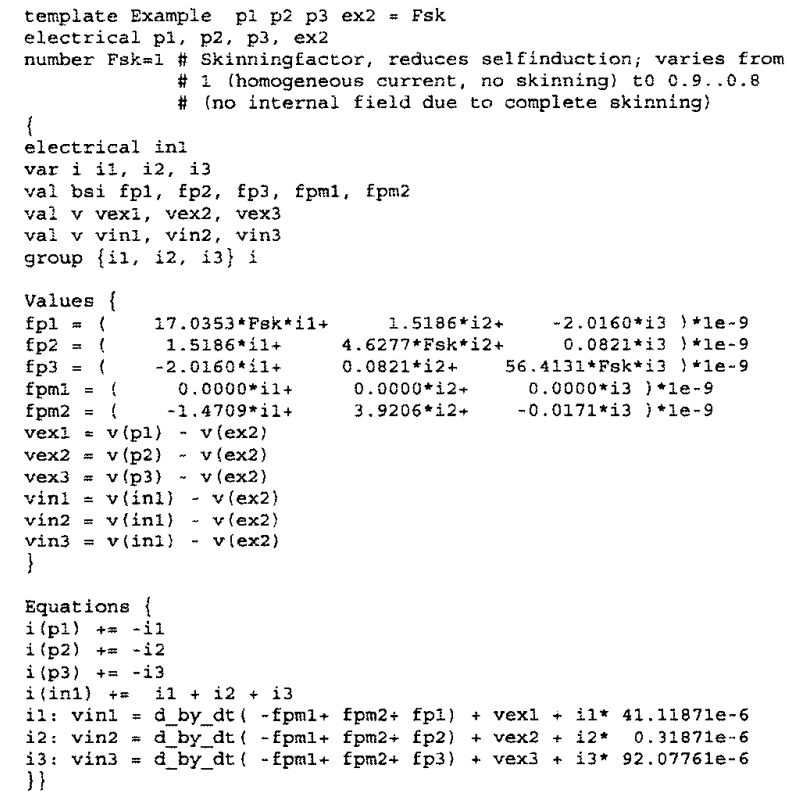

Fig. 18. Saber template of wiring model

\section{VALIDATION OF THE MODEL}

A $2.5 \mathrm{kV} / 2 \mathrm{kA}$ inverterleg according to Fig. 19 was used to check the validity of the modeling. The physical representation of the circuit is depicted in Fig. 20. The wiremodel is given in Fig. 21.

Fig. 23 gives the results, each figure presenting three waveforms. The first one is the simulation with the model developed in this paper. The second one is the measurement. The third one is a simulated waveform without parasitics; these waveforms can be obtained by simulating the circuit of Fig. 19 without any parasitic addition.

In comparing the waveform with and the one without parasitics, one gets an idea of how this model adds to the conventional approach. In comparing the simulation with the measurement one sees in how far this addition is correct. 


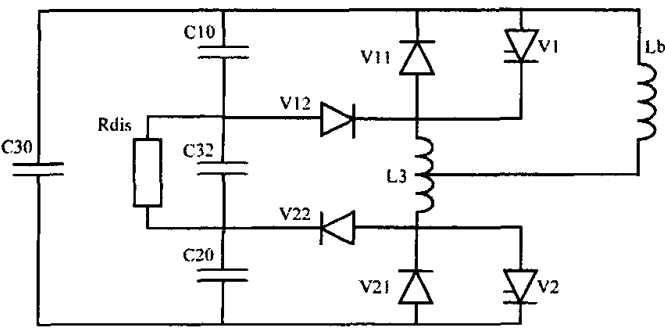

Fig. 19. Circuit used for validation modeling

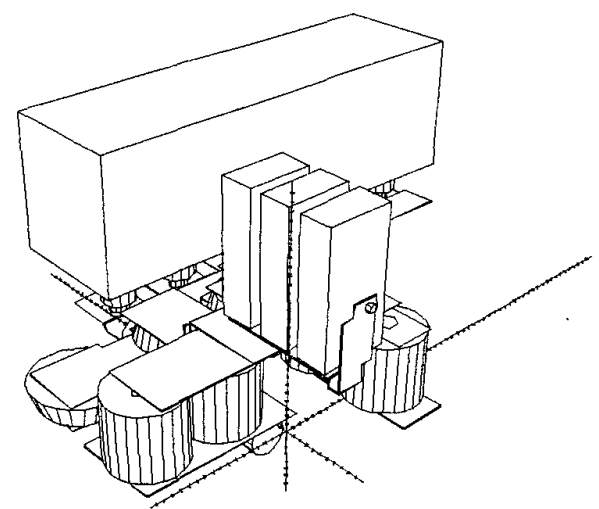

Fig. 20. Physical 3D drawing of circuit used for validation modeling

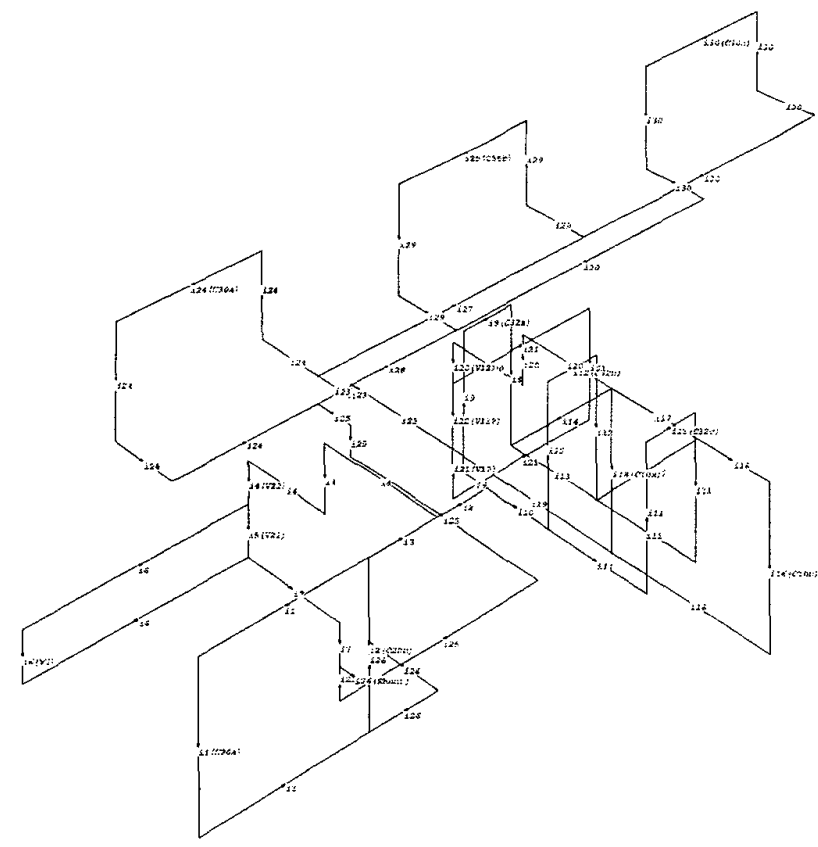

Fig. 21. wire-model of circuit: centre lines of currents in wiring and components
The time-axes is for all the waveforms the same en runs from $t=0 \mu$ s untill $t=200 \mu s$; some waveforms only depict a part of this axes. With reference to Fig. 23 three intervals are defined.

- Interval 1 runs from $t=20 \mu$ s untill $t=120 \mu$ s

- Interval 2 runs from $n t=125 \mu$ s untill $t=155 \mu$ s.

- Interval 3 runs from $t=155 \mu$ s untill $t=200 \mu s$.

Outside these regions the parasitic inductances hardly play any role. This is due to the fact that outside these regions currents and voltage vary only slowly.

Initially (at $t=0$ ) the circuit is in rest: capacitor C30 is charged up to $2 \mathrm{kV}$ and the current in the load $\mathrm{Lb}$ is zero. GTO's V1 and V2 are both blocking. GTO V1 remains blocking during the whole experiment, whereas GTO V1 is brought into conduction at $t=5 \mu \mathrm{s}$ and switched off at $\mathfrak{t}=127 \mu \mathrm{s}$. During conduction-time of V2 (from $t=5 \mu \mathrm{s}$ untillt $=127 \mu \mathrm{s}$ ), a current builds up in $\mathrm{Lb}$ reaching a maximum value of $520 \mathrm{~A}$, shortly after the moment of switching off $\mathrm{V} 2$.

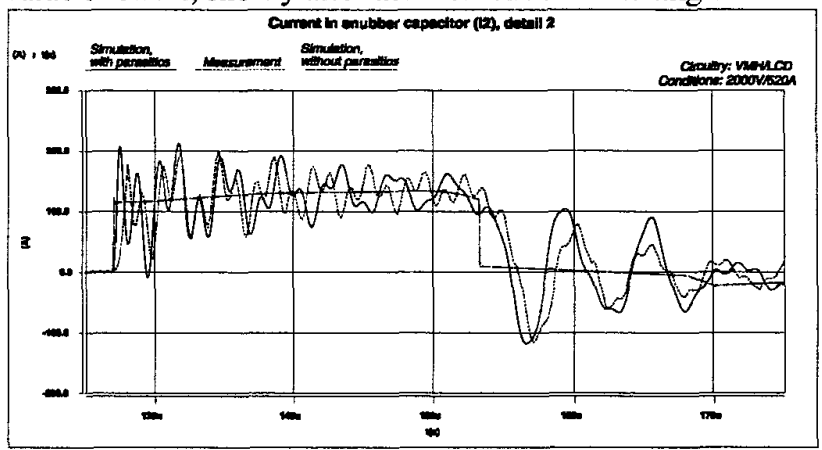

Fig. 22. Simulation with parasitics calcuted with Fsk $=1$

Consider Fig. 23. It turns out that the agreement between measurement and simulation is on interval 2 considerably better than on the intervals 1 and 3 . Especially the damping disagrees on these two intervals. A closer enquiry into this matter reveals that on interval 2 none of the semiconductors carries a high-frequent current. This implies that the calculation of currents on this interval is substantially less affected by the modeling of the semiconductors than eisewhere. Especially interval 1 exhibits a repeated reverse and forward recovery of diode V12. The losses connected with this phenomenon may have significant impact on the damping. It can be concluded that a good to reasonable agreement occurs when the modeling of the wiring is to a large extent responsible for the simulation results.

The waveshapes of Fig. 23 have been obtained by setting Fsk to 0.85 . To get an idea of the influence of this factor, one of the graphs of Fig. 23 is given for Fsk $=1$ in Fig. 22. The differences are only small.

Non-homogeneous current can of course be incorporated in the circuit-simulation by subdividing each current-block into several sub-blocks. In the simulation it will appear that the outer blocks have more current than the inner ones. This method however increases the size of the 
template greatly. It is the authors object to develop a more smart way of dealing with skinning in the nearby future.

Apart from the ultimate template the program generates intermediate results as matrices of inductive coupling between all blocks and branches. These can be very useful as well in establishing the connection between geometrical dimension and the electrical behaviour of the circuit.

\section{Conclusion}

A Method of modeling parasitic inductances has been developed from the theoretical basis to the implementation and validation. Measurement results show that the modeling is accurate and facilitate a prior assessment of geometrical features of a circuit. The rather crude approach of approximating the circuit by homogeneous current blocks already gives very good results. The influence of the factor Fsk suggests that a refinement of this model especially on the point of modeling the sefinductance, may even further improve accuracy.

\section{Literature}

[1] Ruehli, A.E., "Inductance Calculations in a Complex Integrated Circuit Environment", IBM Journal Research Development, september 1972.

[2] Hoer, C. and Love, C., "Exact Inductance Equations for Rectangular Conductors With Applications to More Complicated Geometries", JOURNAL OF RESEARCH of the National Bureau of Standards-C. Engineering and Instrumentation Vol. 69C, No.2, April-June 1965
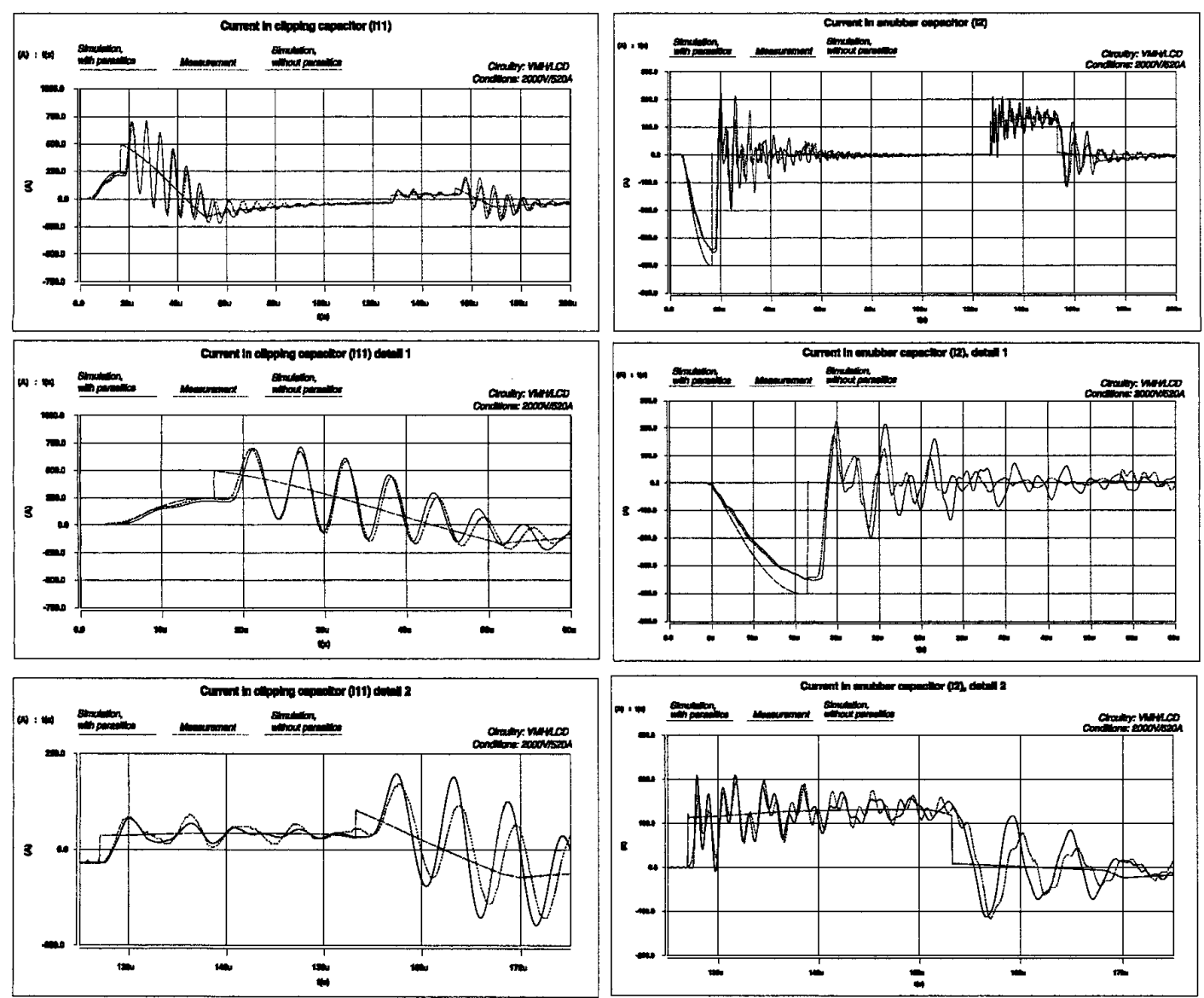

Fig. 23. Measurement and simulation results 